\title{
Accelerated 3D numerical scheme for the evaluation of hysteresis in ferromagnetic grains
}

\author{
Ben Van de Wiele ${ }^{1}$, Luc Dupré ${ }^{1}$, and Femke Olyslager ${ }^{2}$ \\ ${ }^{1}$ Department of Electrical Energy, Systems and Automation, \\ ${ }^{2}$ Department of Information Technology, \\ Ghent University, Sint-Pietersnieuwstraat 41, B-9000 Ghent, Belgium. \\ ben.vandewiele@ugent.be
}

\begin{abstract}
The magnetic behavior of iron based ferromagnetic materials is important for the improvement of performances of electromagnetic devices. This paper deals with an accelerated 3D model that computes the magnetic behavior of an iron grain under a varying applied field starting from its microscopic material parameters. The dynamics of the magnetic dipoles $M$ in each space point of the grain are computed by integrating the Landau-Lifshitz equation using a semi-analytical predictorcorrector time stepping scheme. In the numerical computations, the spatial discretization is obtained by finite different techniques. Evaluation of the magnetostatic field is done using Fast Fourier Transforms. This is the most time consuming part of the computations and in this paper we will demonstrate that this can be accelerated by computing the magnetostatic field on a higher discretization level. The values of the magnetostatic field in each finite difference cell are determined through interpolation, still preserving a very good precision.
\end{abstract}

\section{INTRODUCTION}

Classically the iron losses in electrical steels are characterized and qualified by means of standards which are based on simple, unidirectional sinusoidal flux excitations using e.g. Epstein, ring core or single sheet measurement equipment. Generally, the measured iron losses in electromagnetic devices exceed the value based on these standard measurements. This is caused by nonuniform phenomena like distorted flux distributions, local rotational excitations, changes in the magnetic characteristics due to the mechanical treatment, etc. Understanding these phenomena inside the electrical steel is an indispensable step in the process of developing electromagnetic devices with lower core losses, resulting in a lower energy consumption. Here, the relation between the electromagnetic behavior and the microstructure of the electrical steel is crucial as the microstructure is directly related to the material production and treatment techniques. Hence, a good model for the magnetic behavior of ferromagnetic materials starts from microstructural features like the presence of lattice effects, grains, stresses, crystal defects, etc.

\section{MiCROMAGNETIC THEORY}

Here we focus on the magnetic behavior of a ferromagnetic - pure iron - grain exposed to a time varying external magnetic field. The relation between the magnetization of the grain and the external applied field is expressed by the hysteresis loop of the grain. The micromagnetic theory describes the magnetic dynamics in the material, based on the microscopic material parameters and the applied field. This theory has been adopted to small storage devices but current available computer resources make it possible to use the micromagnetic theory also in models for larger 3D structures as an iron grain. The micromagnetic theory of ferromagnetic materials is based on the assumption, following Landau and Lifshitz [1], that the magnetization of magnetic dipoles $M$ varies with the position, but that it has a fixed temperature dependent magnitude $|\mathbf{M}|=$ $M_{s}$ (below Curie-temperature). The evolution of $\mathbf{M}=M_{s} \mathbf{m}$ is governed by the Landau-Lifshitz-equation (LL-equation)

$$
\frac{\partial \mathbf{m}}{\partial t}=\frac{\gamma_{G}}{1+\alpha^{2}} \mathbf{m} \times \mathbf{H}_{e f f}+\frac{\alpha \gamma_{G}}{1+\alpha^{2}} \mathbf{m} \times\left(\mathbf{m} \times \mathbf{H}_{e f f}\right)
$$

with $\alpha$ and $\gamma_{G}$ the damping constant and gyromagnetic constant respectively. This equation is an extension of the static micromagnetic equilibrium condition $\mathbf{m}(\mathbf{r}) \times \mathbf{H}_{\text {eff }}(\mathbf{r})=0$. Here, and in the LL-equation (1), the influence of the microscopic material parameters and the applied field is included through the effective field which contains the applied field, the exchange field, the anisotropy field, the magnetostatic field and the magnetoelastic field.

\section{3D NUMERICAL SCHEME}

In the 3D numerical scheme, the grain is discretized in cubical finite difference (FD) cells. Each FD cell contains one magnetic dipole $\mathbf{M}$ located in the center of the cell with fixed amplitude $M_{s}$ and varying orientation. The quasi-static applied magnetic field $\mathbf{H}_{a}$ is approximated by a piecewise constant time function. It is assumed that at the moment the applied field $\mathbf{H}_{a}$ jumps from a constant value to the next one, the material is in static micromagnetic equilibrium. Using the LLequation (1), the magnetization dynamics in each FD cell is computed by time stepping until a new static micromagnetic equilibrium is obtained corresponding to the new value for the applied field. This time stepping is performed by a time and memory efficient semi-analytical predictor corrector time stepping scheme [2]. In this time stepping scheme, the optimal discretization length of the FD cell is $9 \mathrm{~nm}$ leading to about $10000000 \mathrm{FD}$ cells for a grain with dimensions of order $\mu \mathrm{m}$.

In the time stepping scheme, the effective field $\mathbf{H}_{\text {eff }}$ has to be evaluated twice in each FD cell for every time step. The most time consuming part in this computation comes from the evaluation of the magnetostatic field $\mathbf{H}_{m s}$ which follows from $\nabla \cdot \mathbf{H}_{m s}=-\nabla \cdot \mathbf{M}$ and $\nabla \times \mathbf{H}_{m s}=0$ using Greens functions:

$$
\mathbf{H}_{m s}=\frac{M_{s}}{4 \pi} \int_{\Omega}\left(\frac{\mathbf{m}}{\left|\mathbf{r}-\mathbf{r}^{\prime}\right|^{3}}-\frac{\left(\mathbf{m} \cdot\left(\mathbf{r}-\mathbf{r}^{\prime}\right)\right)\left(\mathbf{r}-\mathbf{r}^{\prime}\right)}{\left|\mathbf{r}-\mathbf{r}^{\prime}\right|^{5}}\right) \mathrm{d} \Omega^{\prime}
$$


with $\Omega$ the volume of the considered grain. The magnetostatic field (2) is computed using Fast Fourier transforms, exploiting its convolution structure $\mathbf{H}_{m s}=\mathbf{g}(\mathbf{r}) \star \mathbf{m}(\mathbf{r})$ with $\mathbf{g}(\mathbf{r})$ a symmetrical tensor containing all geometrical information. The convolution product is then performed by (i) Fourier transforming the data $\left.\mathbf{g}_{(} \mathbf{r}\right)$ once in the set up phase of the algorithm and Fourier transforming $\mathbf{m}(\mathbf{r})$ every time step, (ii) multiplying the Fourier transformed data point by point and (iii) inverse Fourier transforming the product. When a grain is considered containing $N$ FD cells, the computational complexity of the Fourier transforms scale as $\mathcal{O}(N \log N)$ and the total algorithm scales as

$$
\sim\left(N \log _{10} N+4.825 N\right) .
$$

For $\mu m$-sized grains most of the CPU time is spent on the calculation of the Fourier transforms needed for the magnetostatic field. This numerical scheme leads to an exact evaluation of the magnetostatic field $\mathbf{H}_{m s}$ in the center of every FD cell.

An approximate computation of the magnetostatic field $\mathbf{H}_{m s}$ can be performed when the magnetostatic field is computed on a higher discretization level, combining $n$ cells in each discretization direction and replacing them by an average magnetization $\tilde{\mathbf{m}}$. The computation of the magnetostatic field on this discretization level $\tilde{\mathbf{H}}_{\mathbf{m s}}$ scales as $\mathcal{O}\left(N / n^{3} \log N / n^{3}\right)$. The magnetostatic field $\mathbf{H}_{m s}$ in each FD cell is then computed by interpolation of the values $\tilde{\mathbf{H}}_{m s}$. In this case the total algorithm scales as

$$
\sim\left(\frac{N}{n^{3}} \log _{10} \frac{N}{n^{3}}+1.98 \frac{N}{n^{3}}+3.76 N\right)
$$

where the term proportional to $N / n^{3}$ contains contributions from the point by point products mentioned above and from the Fourier transforms themselves.

\section{NUMERICAL RESULTS}

The validity of the approximate computation of the magnetostatic field $\mathbf{H}_{m s}$ hysteresis loops is evaluated for a cuboid grain of size $1.08 \mu m \times 4.32 \mu m \times 1.08 \mu m$. The external magnetic field is applied along the longest edge of the grain and the magnetostatic field is computed on different discretization levels $(n=1,2, \ldots, 5)$. The applied field $\mathbf{H}_{a}$ is described with $\# H_{a}=400$ constant values, leading to an average of 10000 time steps to compute the hysteresis loop. Table I shows the CPU time needed for one time step and the precision achieved for different $n$. The precision is defined relative to the hysteresis loop computed without interpolation $(n=1)$ :

$$
\text { precision }=\frac{1}{\# H_{a}} \sum_{i=1}^{\# H_{a}}\left|<\mathbf{m}_{n \neq 1}>_{i}-<\mathbf{m}_{n=1}>_{i}\right|
$$

with $<\mathbf{m}_{n}>_{i}$ the average magnetization of the grain when $\# H_{a}=i$. The CPU time decreases drastically for $n=2$ and $n=3$, for higher $n$ the term proportional to $N$ dominates in (4). All loops for $n<5$ have a similar good precision of $2 \%$. The hysteresis loops for $n=1$ and $n=3$ are shown in Fig. 1(a), while Fig. 1(b) shows the difference between the two descending branches of the loops. The peaks in the curve correspond to jumps in the hysteresis loops taken at slightly different values of the applied field.

\section{CONCLUSION}

One concludes that the evaluation of the magnetostatic field $\mathbf{H}_{m s}$ on a discretization level corresponding to $n=3$ reduces the CPU time with about $70 \%$ still keeping a very good precision with respect to the exact computations.

TABLE I

PRECISION AND CPU TIME (ONE TIME STEP) FOR DIFFERENT LOOPS

\begin{tabular}{ccc}
\hline$n$ & CPU time $[s]$ & precision [\%] \\
\hline 1 & 36.96 & - \\
2 & 13.54 & 2.01 \\
3 & 10.98 & 1.91 \\
4 & 10.76 & 1.96 \\
5 & 10.05 & 2.54 \\
\hline
\end{tabular}

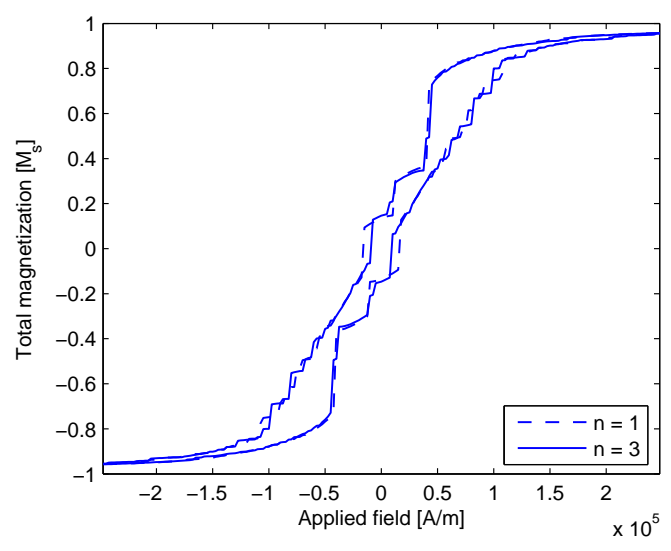

(a) hysteresis loops

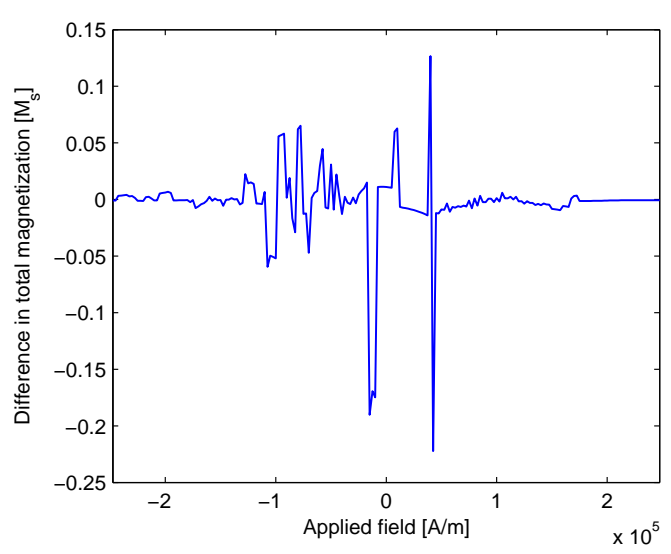

(b) difference in magnetization

Fig. 1. Hysteresis loops for a cuboid iron grain of size $1.08 \mu \mathrm{m} \times 4.32 \mu \mathrm{m} \times$ $1.08 \mu \mathrm{m}$

This work was supported by the Institute for the Promotion of Innovation through Science and Technology in Flanders (IWT-Vlaanderen) SB/51032.

\section{REFERENCES}

[1] L. D. Landau and E. M. Lifshitz, Electrodynamics of continiuous media, Pergamom Press X: Oxford-London-New York-Paris, 1960.

[2] B. Van de Wiele, F. Olyslager and L. Dupré, "Fast numerical 3D-scheme for the simulation of hysteresis in ferromagnetic grains", Journal of Applied Physics, submitted for publication. 


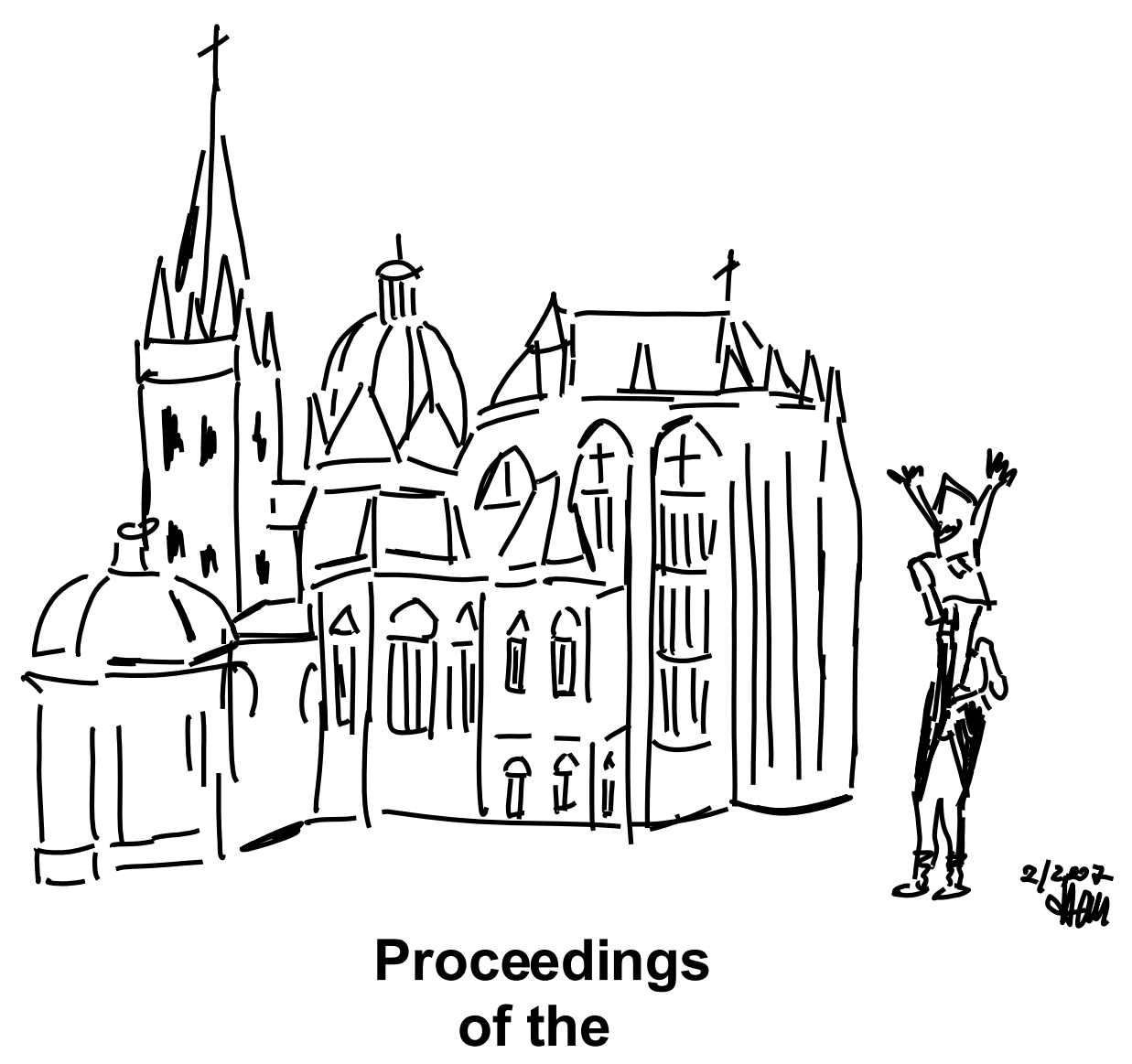

$16^{\text {th }}$ Conference on the Computation of Electromagnetic Fields

\section{COMPUMAG 2007}

ITG INFORMATIONSTEGHNISGHE GESELLSCHAFT IM VDE

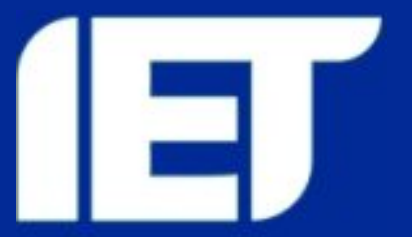

The Knowledge Network
June $24^{\text {th }}-28^{\text {th }}$

Aachen, Germany 